\title{
Comparison of Intraocular Pressure and Anterior Chamber Angle Changes between Pilocarpine and Laser Peripheral Iridotomy
}

\author{
Ardiella Yunard ${ }^{1}$, Virna D Oktariana ${ }^{2}$, Widya Artini ${ }^{3}$, Joedo Prihartono ${ }^{4}$
}

\begin{abstract}
Aim: To compare intraocular pressure and anterior chamber angle changes between pilocarpine and laser peripheral iridotomy in primary angle closure.

Materials and methods: In this clinical trial study, 34 eyes of 29 patients with primary angle-closure were prospectively enrolled between November 2015 and February 2016. Intraocular pressure and anterior segment optical coherence tomography were performed at three separate times: on the initial conditions, 3-5 days of administration of topical pilocarpine $2 \%$, and 1 week after laser iridotomy. Anterior chamber angle parameters were the angle opening distance (AOD) and trabecular-iris space area (TISA).

Results: The intraocular pressure reduction following pilocarpine administration was significant compared to laser iridotomy: $3.9 \mathrm{~mm} \mathrm{Hg}(-32.5$ to 0.20 ) vs $1.8 \mathrm{~mm} \mathrm{Hg}$ ( -33.5 to 2.30) ( $p=0.002$ ). Meanwhile, the increment of angle parameters following laser iridotomy was significant compared to pilocarpine. The AOD750 increment of both nasal and temporal quadrant following laser iridotomy was significant compared to pilocarpine: $0.13 \mathrm{~mm}(-0.27$ to 0.28$)$ vs $0.05 \mathrm{~mm}(-0.35$ to 0.29$)(p=0.003)$ and $0.12 \mathrm{~mm}(-0.10$ to 0.34$)$ vs $0.04 \mathrm{~mm}(-0.27$ to 0.19$)(p=0.002)$, respectively. The TISA750 increment of both nasal and temporal quadrant following laser iridotomy was also significant compared to pilocarpine: $0.05 \mathrm{~mm}^{2}$ $(-0.06$ to 0.20$)$ vs $0.02 \mathrm{~mm}^{2}$ ( -0.12 to 0.13$)(p=0.023)$ and $0.04 \mathrm{~mm}^{2}(-0.04$ to 0.17$)$ vs $0.01 \mathrm{~mm}^{2}(-0.14$ to 0.18$)(p=0.012)$, respectively.

Conclusion: Laser peripheral iridotomy widens the angle greater than topical pilocarpine, but topical pilocarpine lowers the intraocular pressure greater than laser iridotomy. These data suggest that pilocarpine has another mechanism to decrease the intraocular pressure in primary angle-closure, besides widening the angle.

Keywords: Anterior chamber angle, Clinical trial, Intraocular pressure, Laser peripheral iridotomy, Pilocarpine.

Journal of Current Glaucoma Practice (2019): 10.5005/jp-journals-10078-1245
\end{abstract}

\section{INTRODUCTION}

Visual impairment in primary angle closure glaucoma is 2-3 times more common compared to primary open angle glaucoma despite the less prevalence of primary angle-closure glaucoma compared to primary open angle glaucoma. ${ }^{1,2}$ Acute attack of angle closure glaucoma causes blindness in $10 \%$ of the total cases. ${ }^{2,3}$ Quigley et al. ${ }^{4}$ predicted the number of patients with bilateral blindness due to angle closure glaucoma in 2020 would reach 5.3 million.

Relative pupillary block is the main mechanism of aqueous flow obstruction in angle closure glaucoma patients. In pupillary block, there is an aqueous flow obstruction from posterior to anterior chamber through pupil, causing higher pressure in posterior chamber than the anterior chamber. This pressure gradient pushes peripheral iris to anterior, closing the anterior chamber angle. ${ }^{5,6}$

Anterior chamber angle evaluation with gonioscopy is the gold standard until now, but it has several limitations such as subjective result, dependency on examiner expertise, anatomical distortion due to pressure effect, and difficulty in quantitative measurement. ${ }^{2,7-9}$

Anterior segment optical coherence tomography (ASOCT) is an imaging technique which is able to give cross-sectional images of the anterior segment with high resolution. The advantages of ASOCT are faster and easier procedure, more comfortable for the patients with no contact involved, less anatomical distortion caused by contact pressure, as well as quantitative and more objective results. ${ }^{2,8,10}$

Therapeutic principle in angle closure is by removing pupillary block. Laser peripheral iridotomy (LPI) is the treatment of choice

\footnotetext{
${ }^{1-3}$ Department of Ophthalmology, Faculty of Medicine, Universitas Indonesia, Cipto Mangunkusumo Hospital, Jakarta, Indonesia

${ }^{4}$ Department of Community Medicine, Faculty of Medicine, Universitas Indonesia, Cipto Mangunkusumo Hospital, Jakarta, Indonesia
}

Corresponding Author: Ardiella Yunard, Department of Ophthalmology, Faculty of Medicine, Universitas Indonesia, Cipto Mangunkusumo Hospital, Jakarta, Indonesia, Phone: +62 8128103081, e-mail:dr.diella@gmail.com

How to cite this article: Yunard A, Oktariana VD, et al. Comparison of Intraocular Pressure and Anterior Chamber Angle Changes between Pilocarpine and Laser Peripheral Iridotomy. J Curr Glaucoma Pract 2019;13(1):32-36.

Source of support: Nil

Conflict of interest: None

by making a hole in iris, allowing aqueous flow shunt from the posterior to anterior chamber and causing convex iris configuration to be more flattened and opening anterior chamber angle. ${ }^{2,8,11,12}$

The use of miotic agents such as pilocarpine in shallow angle is still currently debated. The rationale for pilocarpine use is to pull peripheral iris away from trabecular meshwork to open the anterior chamber angle. However, pilocarpine can also cause closure of the angle through the anterior shifting of iris-lens diaphragm due to ciliary muscle contraction and worsens pupillary block. ${ }^{13}$ According to Edwards et al., ${ }^{14}$ pilocarpine gives protective effect to the fellow eye in patients with acute attack. Within a mean period of 5 days 
after acute attack, the probability of acute attack on the fellow eye is $3.3 \%$ when using pilocarpine eye drops. Meanwhile, if it is not given any therapy, the probability of acute attack in the fellow eye is $20 \% .{ }^{14}$ Pilocarpine indication nowadays is as a prophylaxis of acute attack in angle closure glaucoma before iridotomy. ${ }^{9,15}$

Laser peripheral iridotomy is the standard first line therapy for angle closure. However, in Indonesia as a developing country, there are many fringe areas without laser facility. Pilocarpine eye drops is still a temporary therapy of choice while waiting for laser peripheral iridotomy to be available. ${ }^{2,6,16}$

The objective of this study was to compare IOP and anterior chamber angle changes between topical pilocarpine and laser peripheral iridotomy.

\section{Materials and Methods}

We conducted this interventional clinical trial in Cipto Mangunkusumo-Kirana Hospital from November 2015 until February 2016. Ethical review clearance test and informed consent were obtained.

\section{Study Participants}

Patients with primary angle-closure were included in this study. If the patient was administered topical pilocarpine before the study, washout procedure for 48 hours was performed. Individuals excluded if they had cup disc ratio $(C D R) \geq 0.8$, previous intraocular laser or surgery, hazy cornea, and any form of secondary angle closure.

Primary angle-closure included in this study were patients diagnosed as primary angle closure suspect (PACS), primary angle closure (PAC), and primary angle-closure glaucoma (PACG). The diagnosis of PACS was made in eyes with iris and trabecular meshwork contact $\geq 180^{\circ}$ on gonioscopy, IOP $\leq 21 \mathrm{~mm} \mathrm{Hg}$, and without presence of peripheral anterior synechiae (PAS). Primary angle closure (PAC) was diagnosed as iris and trabecular meshwork contact $\geq 180^{\circ}$ on gonioscopy, with either IOP $>21 \mathrm{~mm} \mathrm{Hg}$, and/ or with the presence of peripheral anterior synechiae (PAS). Meanwhile, the diagnosis of PACG was made in eyes with iris and trabecular meshwork contact $\geq 180^{\circ}$ on gonioscopy plus evidence of glaucomatous damage to optic disc and visual field.

The patients were recruited consecutively. If both eyes met the criteria, both were included, with a 1 week interlude between them.

Each patient underwent a standardized ophthalmic examination that included fundus examination, visual acuity determination with a Snellen chart, IOP measurement using Goldmann applanation tonometry, gonioscopy with 4-mirror lens, and ASOCT examination (Cirrus; Carl Zeiss). Examinations were performed at three separate times: at initial baseline visit, 3-5 days following topical pilocarpine administration, and 1 week following laser peripheral iridotomy.

\section{Topical Pilocarpin (2\%)}

The patients were prescribed pilocarpine $2 \%$ eyedrops after initial baseline measurement. They were instructed to administer the eyedrops 4 times a day for 3-5 days. Following administration of topical pilocarpine, the patients underwent measurements before laser procedure.

\section{Laser Peripheral Iridotomy (LPI)}

Laser peripheral iridotomy was performed in the superior region of the iris (10-o'clock to 2-o'clock position) using topical anesthesia with sequential argon and $\mathrm{Nd}$ :Yag lasers. It was performed by two glaucoma consultants (VD and WA) under miotic condition of the pupil following administration of topical pilocarpine $2 \%$ for 3-5 days. Prednisolone acetate $1 \%$ eyedrops were prescribed 4 times a day for a week after procedure. Evaluation was done a week after LPI.

\section{Measurements of Anterior Chamber Angle}

Using Cirrus OCT, two anterior chamber angle parameters (AOD750 and TISA750) were measured. It was performed by one operator for all cases. Angle opening distance was calculated as the perpendicular distance measured from the trabecular meshwork at $750 \mu \mathrm{m}$ anterior to the scleral spur to the anterior iris surface (AOD750). Trabecular-iris space area was calculated as an area bounded anteriorly by the AOD750, posteriorly by a line drawn from the scleral spur perpendicular to the plane of the inner scleral wall to the opposing iris, superiorly by the inner corneoscleral wall, and inferiorly by the iris surface (TISA750). Measurements were taken at both nasal and temporal quadrants.

\section{Statistical Analysis}

The primary outcome of this study was anterior chamber angle parameters and IOP. Statistical analysis was done using SPSS 21.0. Wilcoxon test was used to compare the change after pilocarpine and laser peripheral iridotomy.

\section{Results}

A total of 34 eyes with angle closure were recruited for the study. No participant had ever dropped out.

Table 1 summarizes the demographic and clinical data of the participants including age, gender, visual acuity, diagnosis group, and amount of PAS in PAC and PACG group. The mean (SD) age was 58.1 (8.9) years (age range, $46-80$ years). Most of the participants were female.

The baseline IOP and angle parameters were not significantly different among diagnosis groups (Table 2). The IOP reduction by pilocarpine was significantly greater than $\operatorname{LPI}(p=0.002)$. Meanwhile, changes of anterior chamber angle post-LPI were significantly greater than post-pilocarpine (Table 3).

Primary angle closure glaucoma had significantly the greatest IOP reduction among other diagnosis group, both post-pilocarpine ( $p=0.048)$ (Table 4$)$ and post-LPI $(p=0.002)$ (Table 5). There were no significant differences of anterior chamber angle changes postpilocarpine and post-LPI among diagnosis groups (Tables 4 and 5).

Table 1: Baseline characteristics

\begin{tabular}{ll}
\hline Variable & \\
\hline $\begin{array}{l}\text { Age (year) } \\
\text { Gender }\end{array}$ & $58.1 \pm 8.9$ \\
Male & 8 \\
Female & 21 \\
BCVA & logMAR 0 (logMAR 0 - \\
Diagnosis & logMAR 0.60) \\
PACS & 16 \\
PAC & 11 \\
PACG & 7 \\
PAS amount (clock hour) & \\
PAC & $0-9$ \\
PACG & $3-11$ \\
\hline
\end{tabular}


Table 2: Baseline intraocular pressure and angle parameters among groups

\begin{tabular}{lllll}
\hline Variable & PACS & PAC & PACG & $p^{*}$ \\
\hline IOP $(\mathrm{mm} \mathrm{Hg})$ & $14.77 \pm 3.71$ & $17.16 \pm 3.29$ & $18.70(16.00-50.50)$ & 0.054 \\
AOD750 $(\mathrm{mm})$ nasal & $0.238 \pm 0.16$ & $0.214 \pm 0.17$ & $0.130(0.08-0.65)$ & 0.635 \\
AOD750 $(\mathrm{mm})$ temporal & $0.260 \pm 0.15$ & $0.246 \pm 0.16$ & $0.140(0.10-0.47)$ & 0.477 \\
TISA750 $\left(\mathrm{mm}^{2}\right)$ nasal & $0.123 \pm 0.07$ & $0.084 \pm 0.07$ & $0.05(0.03-0.21)$ & 0.327 \\
TISA750 $\left(\mathrm{mm}^{2}\right)$ temporal & $0.141 \pm 0.06$ & $0.110 \pm 0.07$ & $0.07(0.02-0.21)$ & 0.087 \\
\hline
\end{tabular}

${ }^{*}$ Kruskal-Wallis test

Table 3: Comparison of IOP and anterior chamber angle changes between pilocarpine and laser peripheral iridotomy

\begin{tabular}{|c|c|c|c|c|c|}
\hline \multirow[b]{2}{*}{ Variable } & \multicolumn{2}{|c|}{ Pilocarpine } & \multicolumn{2}{|c|}{ Laser peripheral iridotomy } & \multirow[b]{2}{*}{$p^{*}$} \\
\hline & Median & Range & Median & Range & \\
\hline$\triangle \mathrm{IOP}$ & -3.90 & -32.5 to 0.20 & -1.80 & -33.5 to 2.30 & 0.002 \\
\hline$\triangle \mathrm{AOD750}$ nasal & 0.05 & -0.35 to 0.29 & 0.13 & -0.27 to 0.28 & 0.003 \\
\hline$\triangle \mathrm{AOD750}$ temporal & 0.04 & -0.27 to 0.19 & 0.12 & -0.10 to 0.34 & 0.002 \\
\hline$\Delta \mathrm{TISA750}$ nasal & 0.02 & -0.12 to 0.13 & 0.05 & -0.06 to 0.20 & 0.023 \\
\hline$\Delta$ TISA750 temporal & 0.01 & -0.14 to 0.18 & 0.04 & -0.04 to 0.17 & 0.012 \\
\hline
\end{tabular}

Wilcoxon rank

Table 4: Comparison of IOP and anterior segment changes after pilocarpine among diagnosis

\begin{tabular}{lllll}
\hline & \multicolumn{2}{l}{$\Delta$ baseline-pilocarpine } & & \\
\cline { 2 - 4 } Variable & PACS & $P A C$ & PACG & $0.048^{\mathrm{a}}$ \\
\hline$\Delta \mathrm{IOP}(\mathrm{mm} \mathrm{Hg})$ & $-2.80(-10.00-0.70)$ & $-4.08 \pm 3.2$ & $-12.17 \pm 10.67$ & $0.803^{\mathrm{a}}$ \\
$\Delta$ AOD750 $(\mathrm{mm})$ nasal & $0.042 \pm 0.11$ & $0.03(-0.25-0.13)$ & $0.07(-0.35-0.19)$ & $0.271^{\mathrm{a}}$ \\
$\Delta$ AOD750 $(\mathrm{mm})$ temporal & $0.007 \pm 0.11$ & $-0.016 \pm 0.12$ & $0.076 \pm 0.08$ & $0.680^{\mathrm{a}}$ \\
$\Delta \mathrm{TISA750}\left(\mathrm{mm}^{2}\right)$ nasal & $0.022 \pm 0.05$ & $0.02(-0.12-0.40)$ & $0.026 \pm 0.06$ & $0.219^{\mathrm{b}}$ \\
\hline TISA750 $\left(\mathrm{mm}^{2}\right)$ temporal & $0.02 \pm 0.05$ & $-0.01 \pm 0.05$ & $0.04 \pm 0.07$ & \\
\hline
\end{tabular}

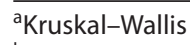

${ }^{\mathrm{b}}$ ANOVA

Table 5: Comparison of IOP and anterior segment changes after laser peripheral iridotomy among diagnosis

\begin{tabular}{|c|c|c|c|c|}
\hline \multirow[b]{2}{*}{ Variable } & \multicolumn{3}{|c|}{$\Delta$ baseline-laser peripheral iridotomy } & \multirow[b]{2}{*}{$p$} \\
\hline & PACS & PAC & PACG & \\
\hline$\Delta \mathrm{IOP}(\mathrm{mm} \mathrm{Hg})$ & $-1.11 \pm 1.87$ & $-2.88 \pm 3.13$ & $-7.40(-33.50$ to -2.00$)$ & $0.002^{\mathrm{a}}$ \\
\hline$\triangle \mathrm{AOD} 750(\mathrm{~mm})$ nasal & $0.119 \pm 0.12$ & $0.14(-0.01$ to 0.17$)$ & $0.021 \pm 0.17$ & $0.205^{\mathrm{a}}$ \\
\hline$\triangle \mathrm{AOD750}(\mathrm{mm}$ temporal & $0.139 \pm 0.14$ & $0.101 \pm 0.11$ & $0.032 \pm 0.04$ & $0.442^{b}$ \\
\hline$\Delta$ TISA750 $\left(\mathrm{mm}^{2}\right)$ nasal & $0.049 \pm 0.06$ & $0.05 \pm 0.03$ & $0.017 \pm 0.06$ & $0.345^{\mathrm{b}}$ \\
\hline$\Delta$ TISA750 $\left(\mathrm{mm}^{2}\right)$ temporal & $0.060 \pm 0.06$ & $0.039 \pm 0.05$ & $0.00(-0.30$ to 0.15$)$ & $0.268^{\mathrm{a}}$ \\
\hline
\end{tabular}

${ }^{a}$ Kruskal-Wallis

${ }^{\text {b} A N O V A}$

\section{Discussion}

Since pupillary block is the main mechanism causing closed angle, the LPI and pilocarpine $2 \%$ eyedrops are able to eliminate pupillary block through different mechanisms to open the angle.

Comparison of anterior chamber angle changes between pilocarpine $2 \%$ eyedrops and LPI was significantly different. Laser peripheral iridotomy widened the angle more than pilocarpine. Similar to this research, Talajic et al. ${ }^{9}$ using pentacam observed that angle changes by LPI were wider and statistically significant compared to pilocarpine.

The change in angle parameters post-pilocarpine was not different among diagnosis groups. This was possible because pilocarpine works through two different mechanisms, pupillary sphincter muscle contraction causing miosis and pulling peripheral iris, and ciliary muscle contraction which causes lens accommodation and iridolenticular shifts to the anterior. The results of these two mechanisms determined the angle alteration. 9,17-19 Besides that, there was a report that age affects angle alterations by pilocarpine. Ciliary muscle response on pilocarpine decreased in accordance with increasing age. ${ }^{15}$

The greatest change in angle parameters of each group postLPI was in the PACS group, followed by PAC, and the least was PAC, although statistically not significant. This was similar to a study by Han et al. ${ }^{20}$ who observed a greater change in PAC than PACG, although not statistically significant. Another study by Ang et al., ${ }^{10}$ divided the group into 2, PACS and PAC in 1 group with PACG 
in another group. There was no difference of angle parameter change post-LPI between the two groups. The greatest change in angle parameters post-LPI was observed in the PACS group in this study because in PACS the iridotrabecular apposition was still reversible. Meanwhile, there was already synechiae in PAC and PACG, making the angle more difficult to be opened. There were also some patients in PAC (5 patients) and PACG group (4 patients) in this study had PAS at the superior quadrant, which was the site of LPI. In PACG, besides synechiae, there was also more damage of trabecular meshwork and optical nerve. Therefore, the angle change in PACG post-LPI was the smallest among other diagnosis groups. $^{21}$

Intraocular pressure post-pilocarpine and LPI in this study showed a significant reduction. Administration of topical pilocarpine $2 \%$ eyedrops gave statistically significant greater IOP reduction compared to $\mathrm{LPI}$. The greater IOP reduction by pilocarpine, which was not in accordance with the angle changes compared to LPI implied other IOP reduction mechanism of pilocarpine. Pilocarpine, a parasympathomimetic agent pulls scleral spur posteriorly, opening intertrabecular space and Schlemm's canal, improving aqueous outflow. ${ }^{17,22,23}$ This mechanism gave greater IOP reduction, aside from opening the angle.

Intraocular pressure change post-pilocarpine 2\% eyedrops and LPI showed the greatest reduction in PACG, followed by PAC and PACS. This was caused by the higher initial IOP of PACG compared to PAC and PACS. In the PACS group, despite having an initial IOP value within the normal range, it still showed a reduction of IOP either post-pilocarpine administration or LPI. This suggested that both pilocarpine $2 \%$ eyedrops and LPI induced an IOP reduction effect. However, it should be noted that in the PACG or PAC group with initial IOP $>21 \mathrm{~mm} \mathrm{Hg}$, antiglaucoma medicine was administered since the beginning of this study until post-LPI measurements were performed. None of the participants in this study got rescue treatment within a 1 week post-LPI follow-up phase.

The strengths of this study were no dropped-out participants, and was the first study comparing the effect of topical pilocarpine and LPI on IOP and anterior chamber angle changes. The limitation of this study was that angle parameters assessed using AS-OCT were only at the nasal and temporal quadrant.

\section{Conclusion}

Both laser peripheral iridotomy and pilocarpine widen the angle and reduce the intraocular pressure of primary angle closure groups. Their effect was decreased in accordance with the severity of the disease. Primary angle-closure suspect showed the greatest result, followed by primary angle closure, and primary angle closure glaucoma was the least. Laser peripheral iridotomy widen the angle more than pilocarpine, meanwhile pilocarpine reduces the intraocular pressure more than laser peripheral iridotomy. This suggests pilocarpine has other mechanism to reduce intraocular pressure in primary angle-closure besides widening the angle.

\section{Clinical Significance}

By assessing the changes in intraocular pressure and anterior chamber angle after pilocarpine administration and laser iridotomy, we can identify the mechanism and response of the eye to both treatments.

\section{Manufacturer Name}

Pilocarpine 2\%: Cendo Carpine 2\% (Cendo Pharmaceutical, Kota Bandung, Jawa Barat 40258, Indonesia).

\section{Patient Consent Form}

Ethics committee approval was obtained.

\section{References}

1. Esmaeili A, Barazandeh B, et al. Assessment of the anterior chamber parameters after laser iridotomy in primary angle close suspect using pentacam and gonioscopy. Int J Ophthalmol 2013;6:680-684. DOI: 10.3980/j.issn.2222-3959.2013.05.25.

2. Friedman DS, Foster PJ, et al. Angle closure and angle-closure glaucoma: what we are doing now and what we will be doing in the future. Clin Exp Ophthalmol 2012;40:381-387. DOI: 10.1111/j.14429071.2012.02774.x.

3. Tham CC, Leung DY, et al. Effects of phacoemulsification vs combined phaco-trabeculectomy on drainage angle status in primary angle closure glaucoma (PACG). J Glaucoma 2010;19:119-123. DOI: 10.1097/ IJG.0b013e31819d5d0c.

4. Quigley HA, Broman AT. The number of people with glaucoma worldwide in 2010 and 2020. Br J Ophthalmol 2006;90:262-267. DOI: 10.1136/bjo.2005.081224.

5. Razeghinejad MR, Kamali-Sarvestani E. The plateau iris component of primary angle closure glaucoma: developmental or acquired. Med Hypotheses 2007;69:95-98. DOI: 10.1016/j.mehy.2006.11. 025.

6. Weinreb RN, Aung T, et al. The pathophysiology and treatment of glaucoma: a review. JAMA 2014;311:1901-1911. DOI: 10.1001/ jama.2014.3192.

7. Kumar RS, Baskaran M, et al. Prevalence of plateau iris in primary angle closure suspects an ultrasound biomicroscopy study. Ophthalmology 2008;115:430-434. DOI: 10.1016/j.ophtha.2007.07.026.

8. Lee KS, Sung KR, et al. Longitudinal changes in anterior segment parameters after laser peripheral iridotomy assessed by anterior segment optical coherence tomography. Invest Ophthalmol Vis Sci 2013;54:3166-3170. DOI: 10.1167/iovs.13-11630.

9. Talajic JC, Lesk MR, et al. Anterior segment changes after pilocarpine and laser iridotomy for primary angle-closure suspects with Scheimpflug photography. J Glaucoma 2013;22:776-779. DOI: 10.1097/IJG.0b013e318259505a.

10. Ang GS, Wells AP. Changes in Caucasian eyes after laser peripheral iridotomy: an anterior segment optical coherence tomography study. Clin Exp Ophthalmol 2010;38:778-785. DOI: 10.1111/j.14429071.2010.02360.x.

11. Grewal SPS, Jain R, et al. Evaluation of anterior segment changes following laser peripheral iridotomy using pentacam scheimpflug imaging system in eyes with primary angle closure. Highlights of Ophthalmology 2010;36:13-14.

12. Thomas R. Glaucoma in developing countries. Indian J Ophthalmol 2012;60:446-450. DOI: 10.4103/0301-4738.100546.

13. Harasymowycz P, Wang J, et al. Angle closure glaucoma. In. Gault J, Vander J. ed. Ophthalmology secrets in color, 4th ed. Philadelphia: Elsevier; 2015. pp. 154-171.

14. Edwards RS. Behaviour of the fellow eye in acute angle-closure glaucoma. Br J Ophthalmol 1982;66:576-579. DOI: 10.1136/ bjo.66.9.576.

15. Kobayashi $\mathrm{H}$, Kobayashi $\mathrm{K}$, et al. Pilocarpine induces an increase in the anterior chamber angular width in eyes with narrow angles. $\mathrm{Br} J$ Ophthalmol 1999;83:553-558. DOI: 10.1136/bjo.83.5.553.

16. Abe H, Kitazawa Y, et al. Guidelines for Glaucoma, 2nd ed. Tokyo: Japan Glaucoma Society; 2006. pp. 25-33. 
17. Johnstone MA. Aqueous humor outflow system overview. In. Stamper RL, Lieberman MF, et al. ed. Becker-Shaffer's Diagnosis and Therapy of the Glaucomas, 8th ed., Elsevier; 2009. pp. 25-42.

18. Bartlett JD, Fiscella RG, et al. Ocular hypotensive drugs. In. Bartlett JD, Jaanus SD. ed. Clinical ocular pharmacology, 2nd ed. United States: Elsevier; 2008. pp. 139-170.

19. Shiroma LO, Costa VP. Parasympatomimetic. In. Shaarawy TM, Sherwood MB, et al. ed. Glaucoma, 2nd ed. London: Elsevier; 2015. pp. 577-582.

20. Han S, Sung KR, et al. Outcomes of laser peripheral iridotomy in angle closure subgroups according to anterior segment optical coherence tomography parameters. Invest Ophthalmol Vis Sci 2014;55:6795-6801. DOI: 10.1167/iovs.14-14714.

21. Nolan $\mathrm{W}$, Foster $\mathrm{P}$, et al. YAG laser iridotomy treatment for primary angle closure in east Asian eyes. Br J Ophthalmol 2000;84:1255-1259. DOI: 10.1136/bjo.84.11.1255.

22. Yang CC, Chou SC, et al. Anterior chamber angles shallowing and intraocular pressure after topical pilocarpine. J Ocul Pharmacol Ther 1997;13:219-224. DOI: 10.1089/jop.1997.13.219.

23. Zimmerman TJ, Wheeler TM. Miotics: side effects and ways to avoid them. Ophthalmology 1982;89:76-80. DOI: 10.1016/s01616420(82)34866-6. 